\title{
Come costruire un Syllabus Learner-centred? \\ Creazione e Validazione di una Rubrica di (Auto)valutazione del Syllabus
}

\section{How to build a Learner-centred Syllabus? Development and Validation of a (Self) Assessment Rubric}

\author{
Anna Serbati*, Sabrina Maniero*, Marcella Bracale ${ }^{* *}$, Silvia Caretta ${ }^{* * \circ}$
}

\section{Riassunto}

L'articolo presenta il processo di costruzione e validazione di una rubrica di (auto)valutazione di un syllabus learner-centred proposta all'interno del progetto MIRES promosso dall'Università dell'Insubria. Il lavoro discute le premesse teoriche e metodologiche, descrive lo strumento e le fasi di validazione realizzate e delinea le prospettive future di ricerca e di pratica.

Parole chiave: syllabus, rubrica, (auto)valutazione, faculty development, istruzione superiore

\begin{abstract}
The paper presents the design and validation process of a rubric to (self)assess a learner-centred syllabus, created in the context of the MIRES project promoted by University of Insubria. The article discusses theoretical and methodological elements, describes the instrument and the validation steps carried out as well as details possible perspectives for research and practice.

Keywords: syllabus, rubric, (self)assessment, faculty development, higher education

\footnotetext{
* Università di Padova.

${ }^{* *}$ Presidio di Qualità, Università dell'Insubria.

${ }^{\circ} \mathrm{L}$ 'impianto e i contenuti del contributo sono stati discussi e progettati in modo condiviso dagli autori. Tuttavia, i par. 1 e 3.1 sono da attribuire ad Anna Serbati; il par. 2 a Marcella Bracale e Silvia Caretta; il par. 3.2 a Sabrina Maniero. Il par. 3.3 è stato redatto congiuntamente da Anna Serbati e Sabrina Maniero. Il par. 4 è stato scritto da tutte le autrici.
}

Excellence and Innovation in Learning and Teaching (ISSNe 2499-507X), 2021, 1

Doi: 10.3280/exioa1-2021oa12067 


\section{Il Syllabus come Strumento di Apprendimento}

Docenti e studenti nell'istruzione superiore hanno a disposizione una molteplicità di strumenti per costruire assieme il percorso di insegnamento e apprendimento.

Un'azione didattica che miri a promuovere apprendimento significativo e duraturo deve interrogarsi sulle strategie da adottare per accompagnare giovani allievi e allieve nel loro percorso di crescita e di sviluppo. Molte sono le parole che possono guidare l'avvio e il sostegno di un percorso didattico centrato sullo studente e sulla studentessa: competenze, risultati di apprendimento attesi, coinvolgimento, ruolo attivo, co-costruzione, consapevolezza. Ma da dove partire? Da dove iniziare a tracciare una guida e un orientamento al cammino che studenti e studentesse percorrono per acquisire conoscenze e competenze?

Nella cassetta degli attrezzi dei docenti c'è uno strumento progettuale e comunicativo molto potente: il syllabus, altresì detto scheda o programma di insegnamento. Esso rappresenta il primo punto di contatto tra docente e studenti e tra disciplina e studenti. Si tratta di un documento informativo completo che fornisce una molteplicità di informazioni importanti, ma al contempo del primo strumento educativo-didattico, che può coinvolgere e motivare allieve ed allievi ad intraprendere un insegnamento e a contestualizzarlo nel più ampio percorso della loro preparazione alla professione futura in esito al corso di studi da loro scelto.

Trattandosi del primo momento comunicativo con studenti e studentesse, consultato prima in asincrono in autonomia dagli allievi, e poi proposto dal docente nella prima lezione, è importante che sia chiaro, completo e motivante.

La letteratura pedagogica sottolinea da tempo l'importanza di spostare la centratura esclusiva di tutte le azioni didattiche dai soli contenuti da trattare al percorso di apprendimento e quindi ai traguardi di competenza che allievi e allieve raggiungeranno. Ciò vale anche per il syllabus, che è lo strumento pensato per "accogliere" studenti e studentesse e accompagnarli nel percorso di un insegnamento o modulo, definendo con chiarezza ruoli, responsabilità e attese nella partnership docente-studenti che si va a stabilire.

Come ricorda Fulmer (2021) $)^{1}$, un syllabus centrato sullo studente non solo fornisce chiare informazioni sui contenuti e sui metodi dell'insegnamento, ma si focalizza altresì sul percorso di apprendimento, fornendo quindi indicazioni su cosa e come gli studenti e le studentesse apprenderanno. Per far sì che esso ricopra questo ruolo educativo, il tono del linguaggio dovrebbe essere quanto più possibile positivo e coinvolgente, indicando con chiarezza gli elementi importanti da sapere, senza concentrarsi su strategie punitive o di penalizzazione.

\footnotetext{
${ }^{1}$ https://www.learningscientists.org/blog/2017/6/18/weekly-digest-64.
} 
Secondo la definizione fornita dal Brigham Young University Center for Teaching and Learning ${ }^{2}$, un learner-centred syllabus si focalizza sui bisogni di studenti e studentesse e sul loro apprendimento e include informazioni che facilitano il loro successo. Nello specifico, l'attenzione è posta sulla descrizione dello scopo del corso e del motivo per cui sia importante rispetto alle competenze della futura professione, sui risultati attesi dell'apprendimento, sulle modalità di insegnamento e apprendimento previste e sulle attività richieste ad allievi ed allieve per imparare quanto proposto. Inoltre, un learner-centred syllabus include informazioni sulla valutazione sommativa e formativa, sui criteri di valutazione, su scadenze e richieste, sui materiali da studiare e consultare.

Bain (2004) attribuisce al syllabus l'aggettivo "promising", sottolineando l'aspetto della promessa, del patto che si stabilisce attraverso questo strumento tra docente e studenti e studentesse, indicando le mete da raggiungere e come ne sarà verificato il raggiungimento $\mathrm{e}$, di conseguenza, responsabilizzando allieve ed allievi rispetto al proprio percorso di apprendimento. L'autore identifica tre sezioni di questo syllabus-promessa. La prima indica le promesse stesse, ovvero quali abilità e competenze questo insegnamento aiuterà loro a sviluppare, a quali domande contribuirà a rispondere, dando loro un chiaro messaggio rispetto all'autoregolazione e controllo dell'apprendimento, che dipende da quanto sapranno cogliere le opportunità offerte. La seconda sezione, infatti, indica cosa si farà per raggiungere $i$ traguardi di apprendimento, quali saranno le risorse messe a disposizione dal docente e le attività richieste. La terza sezione, da ultimo, precisa invece come studenti e docente apprezzeranno la natura dell'apprendimento e quindi il raggiungimento dei risultati attesi; ciò include le procedure di attribuzione del voto, ma va molto oltre, sottolineando il ruolo della valutazione come strumento a sostegno dell'imparare e incoraggiando studenti e studentesse a massimizzare il loro impegno in questa direzione (Grion \& Serbati, 2019).

La vera sfida per i docenti è riuscire ad interpretare il syllabus come un vero strumento didattico, da un lato di comunicazione con studenti e studentesse e, dall'altro, di progettazione approfondita sul percorso di insegnamento e apprendimento che si accingono ad intraprendere (Robasto, 2018; Serbati, 2019). Ciò richiede un ripensamento dello strumento che vada oltre l'idea di un adempimento formale di tipo burocratico, in direzione di una vera e propria risorsa per "partire con il piede giusto" e per attivare un'azione di riflessione costante ${ }^{3}$.

\footnotetext{
${ }^{2} \mathrm{https://ctl.byu.edu/sites/default/files/designing-a-course-syllabus} \mathrm{0.pdf.}$

${ }^{3}$ Un primo importante contributo per valorizzare il ruolo del syllabus nella didattica è stato offerto dal progetto MIRA - Miglioramento Risultati di Apprendimento: dalla formulazione di obiettivi formativi e risultati di apprendimento alla scelta delle metodologie didattiche e di verifica realizzato dal Presidio Qualità di Ateneo dell'Università
} 
Per fare ciò è necessario offrire ai docenti un supporto costante, che includa azioni formative e di faculty development sul tema della progettazione formativa attraverso la preparazione del syllabus, ma è altresì importante fornire strumenti di guida e di sostegno alla costruzione e all'autovalutazione del syllabus stesso in chiave learner centred.

Il presente lavoro trae ispirazione dallo studio proposto da Palmer, Bach e Streifer (2014) intitolato "Measuring the promise: a learning-focused syllabus rubric": gli Autori propongono la costruzione e validazione di una rubrica di valutazione del syllabus che ne misura l'orientamento learner centred, indipendentemente dalla tipologia di insegnamento e dalla disciplina. La portata di uno strumento di questo tipo è stata colta dalle ricercatrici che hanno pensato di agire, in linea con gli Autori, in direzione della costruzione di una rubrica di valutazione del syllabus calata però nelle peculiarità del contesto italiano. L'obiettivo della costruzione di tale strumento è quello da un lato di supportare azioni di valutazione dell'impatto delle azioni di faculty development e/o di quality assurance attraverso una misura completa e rigorosa che tracci una variazione dei syllabi nel tempo. Dall'altro lato, ancora più importante, lo scopo della proposta della rubrica è quello di accompagnare i docenti nella redazione e autovalutazione del proprio syllabus, in direzione di un'offerta di formazione e supporto alla preparazione e all'uso delle schede insegnamento per mettere studenti e studentesse al centro del loro percorso di apprendimento. Come dimostrato dagli Autori stessi in un successivo lavoro (Palmer, Wheeler \& Aneece, 2016), le percezioni degli allievi ed allieve nel leggere un learningfocused syllabus sono significativamente migliori rispetto ad un syllabus centrato solo sul contenuto, sia per quanto concerne il documento stesso, l'insegnamento descritto e anche il docente. Secondo la loro ricerca, infatti, gli studenti hanno percepito il syllabus come strumento utile per organizzare il proprio studio, l'insegnamento come percorso interessante, rilevante e rigoroso di sostegno all'apprendimento e il docente come supportivo e autenticamente interessato al loro sviluppo.

Concordando con gli Autori che un syllabus centrato sullo studente possa sostenere la motivazione e il coinvolgimento di allieve ed allievi, il presente lavoro descrive la rubrica costruita e il processo di validazione realizzato.

di Trento nel 2016-2018 con la collaborazione e la guida di Anna Serbati: https://www.unitn.it/ateneo/58313/documentazione. 


\section{Il Progetto MIRES promosso dall'Università degli Studi dell'In- subria: Azioni di Accompagnamento alla Stesura del Syllabus e Costruzione della Rubrica di Valutazione}

Nel quadro delle azioni rivolte alla promozione dell'Assicurazione della Qualità, nel 2020, l'Università degli Studi dell'Insubria ha avviato un progetto, non ancora concluso, per il Miglioramento della Redazione dei Syllabi degli insegnamenti (Progetto MIRES), rivolto a tutti i docenti che svolgono attività didattica nei Corsi di Studio dell'Ateneo.

Il progetto, sviluppato dal Presidio della Qualità di Ateneo, intende proporre ai docenti una riflessione sul valore del syllabus come proiezione ragionata dell'intero percorso didattico e come strumento centrale sia per la progettazione e la descrizione di una didattica coerente con la prospettiva studentcentered, sia per il coinvolgimento degli studenti in un patto dialettico sul loro percorso di apprendimento, responsabilizzazione e crescita personale.

Inoltre, in una seconda traiettoria di riflessione, il progetto vuole richiamare l'attenzione sull'utilizzo dei syllabi come parametri di analisi comparativa tra insegnamenti simili e come strumenti di valutazione condivisa del livello di coerenza e di raccordo fra la progettazione delle competenze e dei risultati di apprendimento attesi dell'intero Corso di Studi (a livello macro) e la definizione delle competenze e dei risultati di apprendimento attesi dei singoli insegnamenti che ne fanno parte (a livello micro), in una visione di insieme che evidenzi la presenza di eventuali lacune (competenze dichiarate dal Corso di Studio - CdS, ma non sviluppate all'interno di qualche insegnamento) oppure ridondanze (competenze sviluppate in un numero inutilmente elevato di insegnamenti) (Serbati \& Zaggia, 2012).

L'articolazione metodologico-organizzativa del progetto ha previsto fasi successive.

Una prima fase ha riguardato la messa a punto di strumenti di lavoro:

- l'aggiornamento delle "Linee guida e indicazioni operative per la redazione del syllabus" predisposte dal Presidio della Qualità di Ateneo nel 2019;

- la definizione di una rubrica di valutazione (descritta nel prossimo paragrafo), che ancorasse strumenti sviluppati a livello internazionale alla struttura adottata dall'Ateneo per la redazione del syllabus.

Una seconda fase ha riguardato la valutazione, sulla base della rubrica precedentemente definita e validata, della qualità redazionale di un campione di tre syllabi per ciascun Corso di Studio dell'Ateneo, diversificati per anno di corso e per aree scientifico-disciplinari, per un totale di 120 syllabi.

Una terza fase ha riguardato l'attività di formazione dei docenti, attraverso la realizzazione di tre incontri (a febbraio 2020, aprile 2020 e aprile 2021, i due ultimi realizzati a distanza per emergenza COVID-19) rivolti specificamente ai 
Presidenti e ai componenti delle Commissioni di Qualità di tutti i Corsi di Studio dell'Ateneo, ma aperti anche a tutti i docenti interessati e al personale tecnico amministrativo (Manager della Qualità) che svolge la propria attività lavorativa presso le segreterie didattiche. Sono state così convolte circa 200 persone, attraverso le quali è stato chiesto a tutti i docenti di strutturare nuovamente il proprio syllabus, ponendo attenzione alla coerenza e correlazione fra traguardi dell'apprendimento, metodologie formative e attività di valutazione, dettagliando, di queste ultime, la forma (verifica scritta, orale, preparazione di un lavoro, di un testo, attività di laboratorio, ecc.), la tipologia e, nel caso di prove multiple, il peso di ciascuna prova ai fini della valutazione finale.

In questa fase del progetto è stato anche attivato un Help Desk allo scopo di fornire supporto personalizzato a tutti i docenti dell'Ateneo nella redazione del proprio syllabus o per chiarire e discutere punti specifici ${ }^{4}$. L'Help Desk, inizialmente previsto da marzo 2020 a dicembre 2020, è stato rinnovato per il 2021. In questa fase, altro materiale è stato fornito ai docenti, fra cui cinque "Syllabus essentials" con esempi di best practices diversificati per le diverse aree didattiche presenti in Ateneo (Biologia; Economia; Fisica, Matematica, Ingegneria; Medicina e Chirurgia; Giurisprudenza).

In una fase ulteriore sono stati coinvolti gli studenti e le studentesse attraverso incontri con i loro rappresentanti nelle Commissioni di Assicurazione della Qualità del Corso di Studio e delle Commissioni Paritetiche Docenti-Studenti, allo scopo di accompagnarli alla familiarizzazione e all'efficace utilizzo dei syllabi in fase di preparazione da parte dei docenti.

Infine, una fase, tuttora in corso, è dedicata alla valutazione dei syllabi expost (dopo la partecipazione dei docenti alla formazione) e alla restituzione di tale valutazione ai docenti in incontri di riflessione e discussione per singolo Corso di Studio, cui partecipano componenti del Presidio della Qualità

\footnotetext{
${ }^{4}$ L'help desk è stato affidato alla dott.ssa Sabrina Maniero, che possiede una formazione di tipo psico-pedagogico, con competenze nella progettazione, conduzione e valutazione di interventi formativi. Le attività svolte nell'help desk hanno riguardato l'analisi dei syllabi in tutte le loro parti, facendo attenzione ad assumere un doppio punto di vista: quello di uno studente e quello di un collega docente. Si è utilizzata la rubrica come strumento fondamentale per la valutazione della completezza e correlazione tra tutte le parti, prendendo come riferimento l'inquadramento teorico condiviso nella formazione dei docenti (par. 3.1). Come esito si è restituito a ciascun docente il proprio syllabus integrato da una serie di feedback relativi sia ai punti di forza riscontrati, che alle aree di miglioramento; dove necessario si sono suggeriti degli esempi per facilitare la comprensione delle osservazioni oppure si sono posti dei quesiti di chiarificazione. Con l'invio del feedback è stata data disponibilità ai docenti di fare eventuali domande via mail. Alla fine è stato chiesto loro di integrare il syllabus e quindi di rispedirlo. In alcuni casi tale processo si è ripetuto più volte.
} 
dell'Ateneo, del Nucleo di Valutazione, della Commissione di Assicurazione della Qualità del Corso di Studi. Ad oggi sono stati valutati e restituiti oltre 330 syllabi. Durante lo sviluppo delle attività, sono state realizzate più volte analisi di verifica dell'impatto del progetto e sono stati chiesti pareri e feedback ai docenti e ai presidenti di CdS per individuare e correggere in itinere eventuali criticità.

Se si volesse tentare un primo bilancio dell'esperienza progettuale, che tuttavia è ancora in corso, si può affermare fin d'ora che l'attività ha portato a un miglioramento sensibile della qualità dei syllabi di tutti i Corsi di Studio dell'Ateneo, ma, cosa più interessante, ha fatto emergere necessità di apportare modifiche all'ordinamento didattico di alcuni Corsi di Studio e ha innescato fra i docenti una maggiore circolazione di idee e riflessioni sui metodi di insegnamento, di apprendimento e di valutazione, che ha anche trovato sinergie con altre attività poste in essere dall'Ateneo per la qualità della Didattica.

\section{Una Rubrica di Valutazione e Autovalutazione del Syllabus: dalla Costruzione alla Validazione}

\subsection{Inquadramento Teorico di Riferimento}

Nell'ambito delle azioni progettuali sopra descritte, è stata predisposta una rubrica di valutazione dei syllabi, con l'obiettivo di aiutare i docenti a determinare quanto e come ciascuna scheda di insegnamento comunichi mete e processi di supporto all'apprendimento, in linea con una filosofia learner centred del syllabus.

Ispirate dal lavoro di Palmer et al. (2014), le ricercatrici hanno proceduto ad individuare le dimensioni oggetto di indagine, non seguendo un approccio presenza-assenza, bensì un approccio più olistico che consideri, per ciascuna componente, una gradualità di raggiungimento di ciascun descrittore in ottica di chiarezza e sostegno all'apprendimento. Non si è ritenuto possibile applicare tout-court la versione proposta e validata dagli Autori, data la differenza del nostro contesto e della nostra didattica rispetto a quello americano; pertanto si è proceduto a costruire dimensioni e indicatori che rispondessero alle esigenze del panorama italiano e del format di syllabi dell'Ateneo dell'Insubria, e di molte altre istituzioni accademiche italiane.

Le scelte metodologiche nella costruzione dello strumento si sostengono con la letteratura sul syllabus e sulla progettazione della didattica per assicurare la validità dello strumento. In particolare, gli approcci ispiratori della rubrica sono stati:

- L'uso della teoria del constructive alignment (Biggs, \& Tang, 2007), un 
approccio che supporta lo sviluppo della coerenza esterna degli insegnamenti, facilitando un loro allineamento con i risultati di apprendimento del $\mathrm{CdS}$ e, a partire da questi, favorisce l'esplicitazione di conoscenze e abilità che ogni insegnamento si attende che gli studenti acquisiscano. Tale approccio sostiene inoltre la coerenza interna tra risultati di apprendimento attesi e le scelte metodologiche e didattiche del docente, aiutando ad armonizzare i metodi di insegnamento e di valutazione adottati, garantendo un'esperienza equilibrata per lo studente (Galliani, Zaggia \& Serbati, 2011).

- La definizione accurata di obiettivi formativi e risultati di apprendimento, che costituiscono il driver che guida l'intera costruzione di un syllabus learner centred. Formulare scopi dell'insegnamento offre al docente l'opportunità di definire la direzione e l'intenzionalità della didattica, il contributo olistico che essa offre alla crescita di allieve e allievi (Palmer et al., 2014) e la connessione con la figura professionale preparata dal corso di studio e con le relative competenze. La definizione di precisi risultati di apprendimento ${ }^{5}$ complementa gli obiettivi didattici, indicando con puntualità mete misurabili che studenti e studentesse sono chiamati a raggiungere, ossia conoscenze e abilità attese al termine del percorso di formazione.

- Il riferimento alla tassonomia di Anderson e Krathwohl (2001). Gli Autori, revisionando la storica e nota tassonomia realizzata da Bloom nel 1956, pongono l'attenzione sui processi di pensiero, classificandoli in modo crescente:

1. ricordare: memorizzazione di informazioni con azioni quali riconoscere, rievocare;

2. comprendere: interpretazione e capacità di esprimere idee anche con rielaborazioni personali, con azioni quali interpretare, esemplificare, classificare, riassumere, inferire, comparare, spiegare;

3. applicare: capacità di applicare concretamente contenuti e informazioni per risolvere situazioni problematiche, con azioni quali eseguire, implementare;

4. analizzare: capacità di scomposizione delle situazioni in elementi minimi, nelle loro interrelazioni e nella loro struttura generale, con azioni quali differenziare, organizzare, attribuire;

5. valutare: capacità di formulare giudizi critici autonomi e motivati, con azioni quali controllare, criticare;

\footnotetext{
${ }^{5}$ Come ricorda Moon (2002, p. 62), il termine "obiettivo" può riferirsi sia ad uno scopo didattico (obiettivo di insegnamento) sia ad un apprendimento atteso (obiettivo di apprendimento). Nel presente lavoro, per distinguere questi due piani complementari sia usa il termine "obiettivo" riferito all'intenzionalità didattica e "risultato" riferito invece a ciò che gli studenti e le studentesse apprenderanno al termine di un corso.
} 
6. creare: capacità di generare qualcosa di nuovo, con azioni quali generare, pianificare, produrre.

È stata scelta tale tassonomia in quanto ritenuta completa e con esplicito riferimento da parte degli autori al suo utilizzo come strumento per l'allineamento della progettazione del curricolo dell'insegnamento, della didattica e della valutazione.

\subsection{Lo Strumento}

Come indicato in letteratura (Castoldi, 2009; Comoglio, 2003; Ellerani, Gentile \& Sacristani Mottinelli, 2007; McTighe \& Wiggins, 2004), una rubrica è uno strumento generale di valutazione della qualità dei prodotti e delle prestazioni in un determinato ambito, "un documento che articola le aspettative per un compito, elencando i criteri o ciò che conta e descrivendo i livelli di qualità da eccellente a scadente" (Andrade \& Du, 2005). I criteri sono disposti in modo che ci sia una riga per ogni criterio e ogni cella in quella riga descrive le caratteristiche di un particolare livello o standard per quel criterio.

Nel progetto descritto nel paragrafo precedente è stata costruita una rubrica poiché era emersa la necessità di avere uno strumento di valutazione (in appendice) che permettesse di raggiungere vari obiettivi: a) esplicitare le dimensioni di indagine attraverso cui analizzare i syllabi, b) rendere disponibile ai valutatori uno strumento che rendesse affidabili le loro rilevazioni, c) proporre e condividere con i docenti che avevano partecipato alla formazione uno strumento di auto-valutazione dei propri syllabi.

Un passaggio importante è stato l'identificazione delle dimensioni della rubrica e, successivamente, degli indicatori. A tal proposito nel panorama italiano due studi sono risultati particolarmente significativi a conferma degli approcci ispiratori scelti e sopradescritti. In primis, quello di Perla \& Vinci (2021) presenta un'analisi comparativa fra syllabi di insegnamento di area pedagogica e disciplinare presso l'Università di Bari ed identifica, quali dimensioni di interesse, il grado di chiarezza e di eterogeneità nella strutturazione documentale di Ateneo, la coerenza interna fra obiettivi formativi e risultati di apprendimento dell'insegnamento con gli obiettivi del corso di studio, la coerenza fra obiettivi formativi e risultati di apprendimento dei diversi insegnamenti all'interno dello stesso corso di studio, l'allineamento fra risultati di apprendimento attesi, metodi di insegnamento, contenuti e modalità di verifica.

In secondo luogo, la ricerca di Biasin \& Serbati (2020), seppure con focus più specifico sulla competenza relazionale e comunicativa nel corso di laurea L-19, sottolinea l'importanza della connessione tra le informazioni contenute nella Scheda Unica Annuale (SUA), con specifico riferimento a obiettivi del 
corso di studio e competenze della professione, e i syllabi, in particolare con i risultati di apprendimento attesi di ciascun insegnamento.

Le dimensioni di indagine della rubrica del presente studio sono state pertanto individuate considerando vari elementi: $i$ tre approcci ispiratori, i contenuti delle "Linee guida e indicazioni operative per la redazione del syllabus" aggiornate nella fase iniziale del progetto, la struttura stessa che l'Ateneo ha previsto per il syllabus. Le voci che lo compongono sono: obiettivi formativi e risultati di apprendimento attesi, prerequisiti, contenuti, metodi didattici, verifica dell'apprendimento, testi, altre informazioni. Tutti questi elementi hanno contribuito ad individuare nove dimensioni di analisi, riportate in Tabella 1.

Tabella 1 - Dimensioni di analisi della rubrica di valutazione

\begin{tabular}{|l|}
\hline Area trasversale \\
\hline Rispondenza alle Linee Guida \\
\hline Tono \\
\hline Chiarezza del linguaggio \\
\hline Obiettivi formativi e risultati di apprendimento attesi \\
\hline Prerequisiti \\
\hline Contenuti \\
\hline Metodi didattici \\
\hline Modalità di verifica dell'apprendimento / attività di valutazione \\
\hline Altre informazioni \\
\hline
\end{tabular}

Le prime dimensioni riguardano un'area definita 'trasversale' che concerne il tono ed il linguaggio utilizzato. Questi aspetti sono stati evidenziati dallo studio di riferimento (Palmer et al., 2014) in cui si evince che i syllabi più efficaci sono quelli che vanno oltre una funzione 'contrattuale' (di patto formativo) tra docente e studente, per invitare gli studenti, attraverso uno stile motivante, a impegnarsi come soggetti attivi del processo di apprendimento.

In questa dimensione trasversale rientra anche il rispetto delle indicazioni presenti nelle Linee Guida, intesa in particolare come completezza delle informazioni richieste. Le dimensioni successive della rubrica prevedono l'analisi delle singole voci che compongono il syllabus e gli elementi di ciascuna voce.

Per dare un senso più concreto di come funziona la rubrica, poniamo l'attenzione sulla dimensione 'obiettivi formativi e risultati di apprendimento attesi $(\mathrm{RdA})^{6}$. Cinque indicatori compongono questa dimensione, riportati in Tabella 2 .

\footnotetext{
${ }^{6}$ Cfr nota 5.
} 
Tabella 2 - indicatori relativi alla dimensione di analisi "obiettivi formativi e risultati di apprendimento

\begin{tabular}{|l|}
\hline $\begin{array}{l}\text { Gli obiettivi formativi sono chiaramente articolati, esprimendo la dire- } \\
\text { zione dell'insegnamento }\end{array}$ \\
\hline Gli obiettivi formativi sono coerenti con gli obiettivi formativi del CdS \\
\hline I RdA sono chiaramente articolati e utilizzano specifici verbi di azione \\
\hline I RdA descrivono con chiarezza le conoscenze e le abilità (Descrittori \\
di Dublino 1 e 2) che gli studenti acquisiranno \\
\hline $\begin{array}{l}\text { I RdA descrivono le eventuali competenze trasversali (descrittori di } \\
\text { Dublino 3-4-5) che gli studenti acquisiranno }\end{array}$ \\
\hline
\end{tabular}

Attraverso la rubrica si osserva quindi se gli obiettivi formativi sono chiaramente articolati, esprimendo la direzione dell'insegnamento. Un ulteriore aspetto considerato, in merito agli obiettivi, è verificare se e come tali obiettivi concorrono in modo coerente alla formazione delle competenze della figura professionale prevista in esito al percorso di studi, collegandosi in modo esplicito a quanto indicato nella Scheda SUA del Corso di Studio (CdS). Tale specifica valutazione non è stata svolta dai valutatori, in quanto è stata assegnata a chi ha la possibilità di conoscere nel dettaglio il corso, riservandola ai responsabili del CdS.

In merito ai RdA, tre indicatori esplicitano gli elementi per una loro elaborazione esaustiva e completa. Si richiede che siano descritti in modo chiaro e articolato, per permettere agli studenti di comprendere quali saranno le conoscenze, le abilità e competenze che ci si aspetta acquisiscano nel percorso, con riferimento sia agli aspetti disciplinari che trasversali. I RdA dovrebbero evitare termini vaghi a favore di specifici verbi d'azione (uso della tassonomia) che esprimono le conoscenze, abilità o le competenze precise da acquisire nel corso.

\subsection{Scoring, Validità e Attendibilità della Rubrica}

A differenza dello studio di Palmer et al. (2014) che ha classificato e pesato le differenti componenti della rubrica a seconda della loro importanza, nel presente lavoro non si è ritenuto opportuno ordinare le diverse dimensioni individuate, che si ritengono equamente rilevanti nel contesto istituzionale e nazionale. Si è pertanto attribuito progressivamente il punteggio 1 in corrispondenza del livello parziale, 2 per quello adeguato e 3 per il punteggio del livello pieno per ciascuno dei criteri individuati (per un tot di 96 punti max), con l'obiettivo di raggiungere una differenza massima di 10 punti (pari a circa il 10\%) e non più di un punto di differenza per ciascun criterio tra valutatori indipendenti nell'uso della rubrica.

Si sono proposti tre step per verificare l'affidabilità tra giudici indipendenti. 
Tabella 3 - fasi di validazione della rubrica

\begin{tabular}{|l|}
\hline Utilizzo in modo indipendente della rubrica da parte delle autrici per \\
valutare 3 syllabi \\
\hline Utilizzo in modo indipendente della rubrica da parte di due membri del \\
Presidio di Qualità, con competenze diverse sull'argomento e con nes- \\
suna conoscenza dello strumento, unitamente ad una delle due autrici \\
dello strumento, su un campione di 50 syllabi scelti casualmente \\
(prima della formazione) \\
\hline Utilizzo in modo indipendente della rubrica da parte di due valutatori \\
(la referente del Presidio e la referente dell'Help desk, coautrice dello \\
strumento) su un campione di 120 syllabi (distinti in corsi singoli, corsi \\
integrati e tirocini)
\end{tabular}

In tutte le fasi, sulla base dei risultati, lo strumento è stato corretto e affinato laddove sono emerse incongruenze e criticità. I giudici hanno confrontato i punteggi ottenuti dalla loro analisi e, ove si siano verificate discrepanze, la discussione ha permesso di evidenziare indicatori poco chiari o soggetti ad interpretazione, che sono stati affinati.

Per allineare anche la formulazione dei feedback da dare ai docenti, ogni valutatore, dopo aver fatto l'analisi sui syllabi, ne condivideva l'esito confrontandosi con l'altro valutatore per verificare eventuali disaccordi e discuterli, prima di inviare un riscontro ai docenti attraverso l'Help Desk. Uno degli scopi principali dell'intero progetto era proprio quello di offrire ai docenti un'esperienza formativa e di riflessione, per cui ogni restituzione dei syllabi non è avvenuta fornendo i punteggi della rubrica, bensì inviando un feedback il più possibile comprensibile, specifico e orientato a sostenere un processo di miglioramento (Nicol, 2011).

A seguito di queste fasi sono stati affinati i 16 indicatori e i relativi 48 descrittori dei livelli, intervenendo sull'uso del linguaggio per descrivere con precisione ciascun livello di prestazione e le sue caratteristiche più rilevanti e qualificanti. Era necessario che ogni descrittore fosse definito in maniera il più possibile univoca e non generica, così da ridurre al minimo le ambiguità interpretative; si è cercato di utilizzare espressioni descrittive e non valutative, oltre ad elaborare livelli che evidenziassero i progressi e i punti di avanzamento, non solo le carenze (Castoldi, 2009; Goggings Selke, 2013).

\section{Utilizzi della Rubrica e Prospettive}

Ai fini del progetto MIRES, il prossimo passaggio previsto è la condivisione della rubrica con i docenti, al termine delle azioni formative ancora in 
corso, allo scopo di offrire loro uno strumento autovalutativo del proprio syllabus e di consolidare una cultura non solo della didattica centrata sull'apprendimento, ma anche della valutazione.

Ai fini dello strumento, si prevede di concludere la validazione ampliando il numero di valutatori, coinvolgendo esperti esterni al team e non coinvolti nel progetto, per verificare la tenuta e la chiarezza di dimensioni, indicatori e livelli e l'eventuale integrazione di aspetti non considerati nella forma attuale che potrebbero risultare rilevanti (ad es. l'aggiornamento nel tempo dei syllabi o la completezza di tutti i campi - temi chiave per gli organi deputati all'assicurazione della qualità).

Si auspica che la rubrica possa costituire uno strumento utile per istituzioni accademiche a livello nazionale in tre direzioni:

- come strumento autovalutativo e di guida per i docenti alla scrittura e alla riflessione sul proprio syllabus;

- come strumento valutativo a disposizione degli organi deputati alla valutazione e monitoraggio dei syllabi (Presidi di Qualità, Commissioni Paritetiche Docenti Studenti, Commissioni di Assicurazione della Qualità dei CdS);

- come strumento di verifica, affiancato ad altri, dell'impatto delle azioni di faculty development promosso dagli Atenei, promuovendo, ad esempio, analisi pre-post nell'ambito di percorsi di formazione dei docenti.

Da ultimo, l'auspicio delle autrici è quello che l'approccio learner centred rappresentato dalla rubrica possa contribuire ad una cultura della didattica centrata su studenti e studentesse, in cui il percorso di insegnamento e apprendimento avvenga davvero in una partnership autentica ed efficace tra docente e allievi.

\section{Acknowledgements}

Le Autrici desiderano ringraziare i componenti del Presidio di Qualità dell'Università dell'Insubria e i docenti partecipanti al progetto e alla formazione proposta, nonché gli studenti che hanno preso parte agli incontri organizzati. Le Autrici esprimono riconoscenza anche nei confronti dei revisori del paper, che hanno offerto importanti suggerimenti migliorativi.

\section{Riferimenti bibliografici}

Anderson, L. W. \& Krathwohl, D.R., et al (2001). A taxonomy for learning, teaching and assessing: A revision of Bloom's taxonomy of educational objectives. New York: Longman 
Andrade, H., \& Du, Y. (2005). Student Perspectives on Rubric-Referenced Assessment. Practical Assessment, Research \& Evaluation, 10(3): 1-11

Bain, K. (2004). What the best college teachers do. Cambridge, Massachusetts: Harvard University Press.

Biasin, C. \& Serbati, A. (2020). La formazione universitaria alla competenza relazionale e comunicativa dell'educatore socio-pedagogico. Evidenze e riflessioni da un'analisi documentale nel Corso di Laurea in Scienze dell'educazione e della formazione dell'Università di Padova. Rivista Italiana di Educazione Familiare, 2, 197-219.

Biggs, J., \& Tang, C. (2007). Teaching for Quality Learning. New York, McGrawHill.

Castoldi, M. (2009). Valutare le competenze. Roma: Carocci.

Comoglio, M. (2003). Insegnare e apprendere con il portfolio. Milano: Fabbri.

Ellerani, P., Gentile, M., \& Sacristani Mottinelli, M. (2007). Valutare a scuola, formare competenze. Torino: SEI.

Galliani, L., Zaggia, C., \& Serbati, A. (2011). Apprendere e valutare competenze all'università. Progettazione e sperimentazione di strumenti nelle lauree magistrali. Lecce: Pensa Multimedia.

Goggings Selke, M.J. (2013). Rubric assessment goes to college: objective, comprehensive evaluation of student work. Lanham, MD: Rowan \& Littlefield.

Grion, V., \& Serbati A. (2019). Valutazione sostenibile e feedback nei contesti universitari. Prospettive emergenti ricerche e pratiche. Lecce: Pensa Multimedia.

McTighe, J., \& Wiggins, G. (2004). Understanding by design. Professional development workbook. Alexandria, VA: Association for Supervision and Curriculum Development.

Nicol, D. (2011). Good designs for written feedback for students. In Svinivki, M. \& McKeachie, W. J. (eds). McKeachie's Teaching Tips: Strategies, Research, and Theory for College and University Teachers, Thirteenth Edition (pp. 108-124). US: Wadsworth, Cengage Learning.

Moon, J. (2002). The Module and Programme Development Handbook: Linking Levels, Learning Outcomes and Assessment. London: Kogan Page.

Palmer, M.S., Bach, D. \& Streifer, A.C. (2014). Measuring the promise: a learningfocused syllabus rubric. To Improve the Academy, 33(1), 14-36.

Palmer, M.S., Wheeler, L.B., \& Aneece, I. (2016). Does the Document Matter? The Evolving Role of Syllabi in Higher Education. Change: The Magazine of Higher Learning, 48(4), 36-47, DOI: 10.1080/00091383.2016.1198186.

Perla, L. \& Vinci, V. (2021). Modellistiche co-epistemologiche per la formazione del docente universitario. Il progetto Prodid Uniba. Excellence and Innovation in Learning and Teaching, 1, Special Issue - Faculty Development e Digital Scholarship: questioni di ricerca nell'istruzione superiore, 11-30.

Robasto, D. (2018). Processi di apprendimento e insegnamento nella didattica universitaria. Tra requisiti di sistema e l'innovazione didattica. Excellence and Innovation in Teaching and Learning. 5(I), 38-57.

Serbati, A. (2019). Come definire i traguardi dell'apprendimento degli studenti: dagli obiettivi educativi alle competenze e Learning Outcomes. In Dipace, A. \& 
Tamborra, V. (Eds.). Insegnare in università. Metodi e strumenti per una didattica efficace (pp. 38-57). Milano: FrancoAngeli.

Serbati, A., \& Zaggia, C. (2012). Allineare le metodologie di insegnamento, apprendimento e valutazione ai learning outcomes: una proposta per i corsi di studio di istruzione superiore. Italian Journal of Educational Research, V, 11-26. 


\begin{tabular}{|c|c|c|c|c|}
\hline \multirow[t]{2}{*}{ DIMENSIONI } & \multirow[t]{2}{*}{ INDICATORI } & \multicolumn{3}{|c|}{ LIVELLI } \\
\hline & & Pieno (livello3) & Adeguato (livello2) & Parziale (livello1) \\
\hline $\begin{array}{l}\text { AREA TRA- } \\
\text { SVERSALE } \\
\text { Linee Guida }\end{array}$ & $\begin{array}{l}\text { Le voci di cui si compone il } \\
\text { syllabus sono compilate in } \\
\text { modo coerente a quanto ri- } \\
\text { chiesto dalle linee guida per } \\
\text { la compilazione }\end{array}$ & $\begin{array}{l}\text { Rispetta tutte le indicazioni } \\
\text { delle linee guida per la com- } \\
\text { pilazione } \\
\text { Sono state compilate tutte le } \\
\text { voci in modo coerente }\end{array}$ & $\begin{array}{l}\text { Rispetta alcune delle in- } \\
\text { dicazioni delle linee } \\
\text { guida per la compila- } \\
\text { zione. } \\
\text { Alcune voci sono com- } \\
\text { pilate in modo generico }\end{array}$ & $\begin{array}{l}\text { Rispetta solo in minima parte } \\
\text { le indicazioni delle linee } \\
\text { guida. Le descrizioni inserite } \\
\text { non sono coerenti con le voci } \\
\text { richieste e/o sono spostate in } \\
\text { altre meno pertinenti }\end{array}$ \\
\hline $\begin{array}{l}\text { TRASVER- } \\
\text { SALE/ } \\
\text { Tono }\end{array}$ & $\begin{array}{l}\text { Il tono del linguaggio utiliz- } \\
\text { zato è positivo, coinvol- } \\
\text { gente, invitante }\end{array}$ & $\begin{array}{l}\text { Il linguaggio è positivo, } \\
\text { coinvolgente, invitante in } \\
\text { tutto il testo del syllabus. } \\
\text { Nel complesso ne emerge un } \\
\text { documento che motiva e for- } \\
\text { nisce una struttura e una } \\
\text { guida chiara per gli studenti }\end{array}$ & $\begin{array}{l}\text { Il linguaggio è asettico, } \\
\text { dà informazioni evi- } \\
\text { tando messaggi moti- } \\
\text { vanti e/o le voci del syl- } \\
\text { labus sono compilate in } \\
\text { modo molto conciso }\end{array}$ & $\begin{array}{l}\text { Il linguaggio è direttivo e } \\
\text { puntualizza elementi secon- } \\
\text { dari } \\
\text { Il focus del documento è su } \\
\text { aspetti 'punitivi', divieti }\end{array}$ \\
\hline $\begin{array}{l}\text { TRASVER- } \\
\text { SALE/ } \\
\text { Chiarezza del } \\
\text { linguaggio }\end{array}$ & $\begin{array}{l}\text { Il linguaggio utilizzato è } \\
\text { chiaro e specifico }\end{array}$ & $\begin{array}{l}\text { Linguaggio chiaro e speci- } \\
\text { fico del dominio scientifico, } \\
\text { comprensibile a chi non è } \\
\text { esperto della materia }\end{array}$ & $\begin{array}{l}\text { Alcune parti del testo } \\
\text { sono chiare, altre parti } \\
\text { del documento presen- } \\
\text { tano elementi non com- } \\
\text { prensibili ai non esperti }\end{array}$ & $\begin{array}{l}\text { Linguaggio complesso, che } \\
\text { inserisce tecnicismi ed } \\
\text { espressioni non comprensi- } \\
\text { bili ai non esperti oppure } \\
\text { espressioni vaghe }\end{array}$ \\
\hline
\end{tabular}

Copyright () FrancoAngeli

This work is released under Creative Commons Attribution - Non-Commercial -

No Derivatives License. For terms and conditions of usage

please see: http://creativecommons.org 


\begin{tabular}{|c|c|c|c|c|}
\hline $\begin{array}{l}\text { OBIETTIVI } \\
\text { FORMATIVI E } \\
\text { RISULTATI DI } \\
\text { APPRENDI- } \\
\text { MENTO (RdA) }\end{array}$ & $\begin{array}{l}\text { Gli obiettivi formativi sono } \\
\text { chiaramente articolati, espri- } \\
\text { mendo la direzione dell'in- } \\
\text { segnamento }\end{array}$ & $\begin{array}{l}\text { Gli obiettivi descrivono con } \\
\text { chiarezza, univocità, specifi- } \\
\text { cità (e completezza) la te- } \\
\text { matica che il docente si pro- } \\
\text { pone di trattare durante il } \\
\text { corso, spiegando la natura ed } \\
\text { il significato del percorso } \\
\text { formativo. }\end{array}$ & $\begin{array}{l}\text { Gli obiettivi descrivono } \\
\text { in maniera vaga la tema- } \\
\text { tica che il docente si } \\
\text { propone di trattare du- } \\
\text { rante il corso }\end{array}$ & $\begin{array}{l}\text { Non sono presenti obiettivi } \\
\text { Oppure } \\
\text { Sono descritti elementi spe- } \\
\text { cifici (argomenti del per- } \\
\text { corso) invece di obiettivi } \\
\text { generali che il docente si } \\
\text { propone di trattare. } \\
\text { La descrizione può risultare } \\
\text { ambigua }\end{array}$ \\
\hline $\begin{array}{l}\text { OBIETTIVI } \\
\text { FORMATIVI } \\
\text { (da affidare ai } \\
\text { responsabili del } \\
\text { CdS) }\end{array}$ & $\begin{array}{l}\text { Gli obiettivi formativi sono } \\
\text { coerenti con gli obiettivi for- } \\
\text { mativi del CdS }\end{array}$ & $\begin{array}{l}\text { Gli obiettivi concorrono in } \\
\text { modo coerente alla forma- } \\
\text { zione delle competenze } \\
\text { della/e figura/e professio- } \\
\text { nale/i prevista/e in esito al } \\
\text { percorso di studi, collegan- } \\
\text { dosi in modo esplicito a } \\
\text { quanto indicato nella Scheda } \\
\text { SUA del CdS. } \\
\text { Viene esplicitato il motivo } \\
\text { per cui l'insegnamento è ne- } \\
\text { cessario nell'economia del } \\
\text { percorso }\end{array}$ & $\begin{array}{l}\text { Gli obiettivi concorrono } \\
\text { in modo coerente alla } \\
\text { formazione delle com- } \\
\text { petenze della/e figura/e } \\
\text { professionale/i previ- } \\
\text { sta/e in esito al percorso } \\
\text { di studi }\end{array}$ & $\begin{array}{l}\text { Gli obiettivi esulano dalle } \\
\text { competenze previste per la } \\
\text { figura professionale in esito } \\
\text { al percorso } \\
\text { (Scheda SUA) }\end{array}$ \\
\hline
\end{tabular}

Copyright () FrancoAngeli

This work is released under Creative Commons Attribution - Non-Commercial -

No Derivatives License. For terms and conditions of usage

please see: http://creativecommons.org 


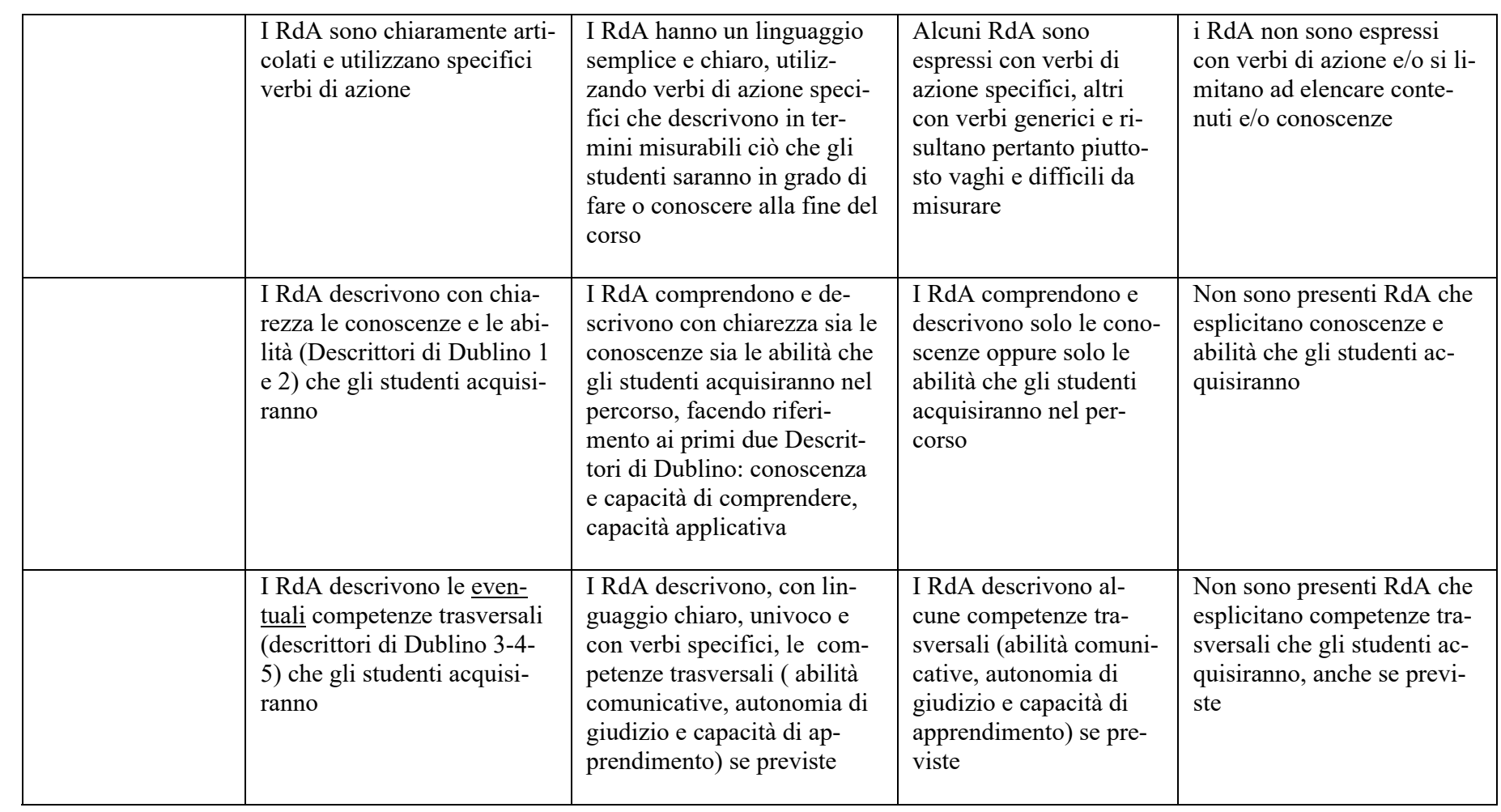

Copyright () FrancoAngeli

This work is released under Creative Commons Attribution - Non-Commercial -

No Derivatives License. For terms and conditions of usage

please see: http://creativecommons.org 


\begin{tabular}{|c|c|c|c|c|}
\hline PREREQUISITI & $\begin{array}{l}\text { Sono presenti eventuali co- } \\
\text { noscenze e abilità ritenute } \\
\text { necessarie per un proficuo } \\
\text { apprendimento }\end{array}$ & $\begin{array}{l}\text { Descrizione chiara e speci- } \\
\text { fica delle conoscenze e abi- } \\
\text { lità ritenute necessarie per } \\
\text { un proficuo apprendimento, } \\
\text { con indicazioni di quali altri } \\
\text { insegnamenti del CdS le pro- } \\
\text { muovano. } \\
\text { Descrizione dei vincoli di } \\
\text { propedeuticità, se obbliga- } \\
\text { tori } \\
\text { Oppure è esplicitato che l'in- } \\
\text { segnamento non prevede } \\
\text { pre-requisiti }\end{array}$ & $\begin{array}{l}\text { Descrizione delle cono- } \\
\text { scenze e abilità ritenute } \\
\text { necessarie per un profi- } \\
\text { cuo apprendimento. } \\
\text { Descrizione dei vincoli } \\
\text { di propedeuticità, se ob- } \\
\text { bligatori }\end{array}$ & $\begin{array}{l}\text { Assenza di indicazioni sui } \\
\text { prerequisiti, che invece } \\
\text { emergono dalla lettura dei } \\
\text { RdA } \\
\text { Oppure non sono richiesti }\end{array}$ \\
\hline CONTENUTI & $\begin{array}{l}\text { I contenuti dell'insegnamento } \\
\text { sono esplicitati in modo sinte- } \\
\text { tico, ma chiaro, indicando i } \\
\text { principali argomenti che ver- } \\
\text { ranno sviluppati }\end{array}$ & $\begin{array}{l}\text { Il syllabus offre un pro- } \\
\text { gramma del corso completa- } \\
\text { mente articolato e logica- } \\
\text { mente sequenziato, chiara- } \\
\text { mente strutturato, per guidare } \\
\text { gli studenti durante il corso, } \\
\text { prevedendo una piena coper- } \\
\text { tura dei RdA. } \\
\text { Indica inoltre chiaramente } \\
\text { quando ulteriori informazioni } \\
\text { verranno fornite in un se- } \\
\text { condo momento }\end{array}$ & $\begin{array}{l}\text { Descrizione chiara e sin- } \\
\text { tetica dei principali argo- } \\
\text { menti trattati, finalizzati, } \\
\text { anche se non esplicita- } \\
\text { mente, al raggiungimento } \\
\text { dei RdA }\end{array}$ & $\begin{array}{l}\text { La descrizione degli argo- } \\
\text { menti copre in parte i RdA } \\
\text { previsti } \\
\text { La descrizione è complicata, } \\
\text { molto lunga oppure troppo } \\
\text { sintetica }\end{array}$ \\
\hline
\end{tabular}

Copyright () FrancoAngeli

This work is released under Creative Commons Attribution - Non-Commercial -

No Derivatives License. For terms and conditions of usage

please see: http://creativecommons.org 


\begin{tabular}{|c|c|c|c|c|}
\hline $\begin{array}{l}\text { METODI DI- } \\
\text { DATTICI }\end{array}$ & $\begin{array}{l}\text { Sono descritti i metodi didat- } \\
\text { tici utilizzati nell'insegna- } \\
\text { mento per facilitare l'appren- } \\
\text { dimento }\end{array}$ & $\begin{array}{l}\text { Descrizione dettagliata e am- } \\
\text { pia dell'organizzazione delle } \\
\text { attività didattiche, sia in ter- } \\
\text { mini di metodi di insegna- } \\
\text { mento (es. lezioni frontali, la- } \\
\text { boratori, animazione di chat e } \\
\text { forum on line...) sia attività } \\
\text { di apprendimento degli stu- } \\
\text { denti (es. ricerca, lavoro di } \\
\text { gruppo, project work, simula- } \\
\text { zioni, attività sul campo, pre- } \\
\text { sentazioni....) }\end{array}$ & $\begin{array}{l}\text { Descrizione dei metodi di } \\
\text { insegnamento usati dal } \\
\text { docente, senza descri- } \\
\text { zione delle attività di ap- } \\
\text { prendimento }\end{array}$ & $\begin{array}{l}\text { Descrizione generica, } \\
\text { breve/sintetica dei metodi di } \\
\text { insegnamento }\end{array}$ \\
\hline $\begin{array}{l}\text { MODALITÀ DI } \\
\text { VERIFICA } \\
\text { DELL'APPREN- } \\
\text { DIMENTO/AT- } \\
\text { TIVITÀ DI VA- } \\
\text { LUTAZIONE }\end{array}$ & $\begin{array}{l}\text { I RdA e le attività di valuta- } \\
\text { zione sono allineati tra loro } \\
\text { (cioè c'è coerenza con RdA, } \\
\text { contenuti, attività didattiche e } \\
\text { attività di valutazione) }\end{array}$ & $\begin{array}{l}\text { Sono esplicite le connessioni } \\
\text { tra i RdA, contenuti, attività } \\
\text { didattiche e le principali atti- } \\
\text { vità di valutazione (vale a } \\
\text { dire, ogni attività di valuta- } \\
\text { zione è associata a uno o più } \\
\text { RdA). Vi è coerenza interna } \\
\text { tra le varie parti }\end{array}$ & $\begin{array}{l}\text { Vi sono le connessioni } \\
\text { tra i RdA, contenuti, atti- } \\
\text { vità didattiche e le princi- } \\
\text { pali attività di valuta- } \\
\text { zione, anche se non espli- } \\
\text { cite }\end{array}$ & $\begin{array}{l}\text { Le attività di valutazione in- } \\
\text { dicate sono sintetiche, non } \\
\text { esplicitano come coprono } \\
\text { tutti i risultati di apprendi- } \\
\text { mento indicati oppure non } \\
\text { sono coerenti con contenuti- } \\
\text { attività didattiche }\end{array}$ \\
\hline
\end{tabular}

Copyright () FrancoAngeli

This work is released under Creative Commons Attribution - Non-Commercial -

No Derivatives License. For terms and conditions of usage

please see: http://creativecommons.org 


\begin{tabular}{|c|c|c|c|c|}
\hline & $\begin{array}{l}\text { Le attività di valutazione } \\
\text { sommativa sono chiaramente } \\
\text { definite }\end{array}$ & $\begin{array}{l}\text { Sono descritte con specificità } \\
\text { le diverse attività di valuta- } \\
\text { zione da fare al termine del } \\
\text { percorso formativo e l'og- } \\
\text { getto di valutazione (tipologie } \\
\text { e strutturazione delle prove) } \\
\text { È riportato il numero e/o fre- } \\
\text { quenza delle prove di valuta- } \\
\text { zione finali } \\
\text { Se presenti più attività di va- } \\
\text { lutazione, viene indicato il } \\
\text { peso assegnato a ciascuna } \\
\text { prova per la valutazione fi- } \\
\text { nale. } \\
\text { Indicazione dei parametri con } \\
\text { cui viene attribuito il voto fi- } \\
\text { nale } \\
\text { Eventuali informazioni anche } \\
\text { per i non frequentanti }\end{array}$ & \begin{tabular}{|l|} 
Sono descritte con speci- \\
ficità le attività di valuta- \\
zione da fare al termine \\
del percorso formativo \\
(tipologie e struttura- \\
zione delle prove) \\
Se presenti più attività di \\
valutazione, viene indi- \\
cato il peso assegnato a \\
ciascuna prova per la va- \\
lutazione finale \\
\\
Rispetto al singolo me- \\
todo di valutazione \\
(prova orale, prova \\
scritta, ecc.) si fa riferi- \\
mento all'oggetto di va- \\
lutazione
\end{tabular} & $\begin{array}{l}\text { Le attività di valutazione in- } \\
\text { dicate fanno riferimento solo } \\
\text { all'esame finale. } \\
\text { È presente un breve elenco di } \\
\text { modalità di valutazione } \\
\text { senza ulteriori specificazioni } \\
\text { Se sono indicate più prove, } \\
\text { potrebbe mancare l'indica- } \\
\text { zione del peso/punteggio as- } \\
\text { segnato }\end{array}$ \\
\hline & $\begin{array}{l}\text { Le attività di valutazione for- } \\
\text { mativa, da svolgere durante } \\
\text { l'insegnamento, sono chiara- } \\
\text { mente definite }\end{array}$ & $\begin{array}{l}\text { Sono indicati vari metodi per } \\
\text { monitorare l'apprendimento } \\
\text { degli studenti durante il per- } \\
\text { corso formativo, specificando } \\
\text { eventuali tempistiche e l'og- } \\
\text { getto di valutazione }\end{array}$ & $\begin{array}{l}\text { È presente una modalità } \\
\text { di monitoraggio e valuta- } \\
\text { zione degli apprendi- } \\
\text { menti durante il percorso } \\
\text { formativo (es. discus- } \\
\text { sioni, dibattiti, fasi di } \\
\text { project work, ecc.) }\end{array}$ & $\begin{array}{l}\text { Sono indicati esclusivamente } \\
\text { metodi di valutazione som- } \\
\text { mativa }\end{array}$ \\
\hline
\end{tabular}

Copyright () FrancoAngeli

This work is released under Creative Commons Attribution - Non-Commercial -

No Derivatives License. For terms and conditions of usage

please see: http://creativecommons.org 


\begin{tabular}{|c|c|c|c|c|}
\hline & $\begin{array}{l}\text { Sono descritti i criteri di valu- } \\
\text { tazione che il docente adot- } \\
\text { terà per valutare le prove }\end{array}$ & $\begin{array}{l}\text { Sono esplicitati in modo } \\
\text { chiaro e specifico i criteri di } \\
\text { valutazione che il docente } \\
\text { adotterà per valutare le prove } \\
\text { che esplicitano cosa il do- } \\
\text { cente 'apprezza', cosa 'valuta } \\
\text { come prestazione di qualità' }\end{array}$ & $\begin{array}{l}\text { Sono indicati alcuni cri- } \\
\text { teri di valutazione che il } \\
\text { docente adotterà per va- } \\
\text { lutare le prove; oppure } \\
\text { criteri generici }\end{array}$ & $\begin{array}{l}\text { I criteri di valutazione non } \\
\text { vengono indicati }\end{array}$ \\
\hline $\begin{array}{l}\text { ALTRE INFOR- } \\
\text { MAZIONI }\end{array}$ & $\begin{array}{l}\text { Sono indicate tutte le infor- } \\
\text { mazioni per poter contattare il } \\
\text { docente (dati per ricevimento) }\end{array}$ & $\begin{array}{l}\text { Sono indicati: e-mail del do- } \\
\text { cente, disponibilità a fissare i } \\
\text { colloqui, sede dove svolgerli. } \\
\text { Sono riportati eventuali giorni } \\
\text { e orari di ricevimento. } \\
\text { Vi sono rimandi a possibilità } \\
\text { di colloqui con il docente in } \\
\text { caso di esigenze specifiche. } \\
\text { Sono presenti eventuali } \\
\text { indicazioni relative alla } \\
\text { possibilità di svolgere tesi su } \\
\text { determinati argomenti e } \\
\text { relative modalità. }\end{array}$ & $\begin{array}{l}\text { Sono indicati: e-mail del } \\
\text { docente e/o numero di te- } \\
\text { lefono }\end{array}$ & $\begin{array}{l}\text { Mancano informazioni rela- } \\
\text { tive al ricevimento del do- } \\
\text { cente } \\
\text { Oppure si fa riferimento ad } \\
\text { altre pagine del sito internet }\end{array}$ \\
\hline
\end{tabular}

Copyright () FrancoAngeli

This work is released under Creative Commons Attribution - Non-Commercial -

No Derivatives License. For terms and conditions of usage

please see: http://creativecommons.org 